\title{
As perspectivas da multimodalidade na análise dos modos e recursos de um aplicativo para o ensino de Inglês
}

Jéssica Rodrigues Esteves;

Berenice Santos Gonçalves

resumo:

As interfaces multimodais permitem uma comunicação mais exploratória e natural, fornecendo aos usuários vários modos de interação. Na educação, a multimodalidade trata do uso de diferentes modos de comunicação para potencializar o processo de ensino-aprendizagem, sobretudo no ensino de línguas estrangeiras. Logo, este artigo se propõe a analisar como a perspectiva da multimodalidade foi adotada na interface de um aplicativo para o ensino de Inglês, considerando a abordagem multimodal no Design de Interfaces e na Semiótica Social. Para tal, foi realizado um estudo de carácter exploratório, com abordagem qualitativa, composto por uma revisão bibliográfica e uma análise descritiva. Os resultados da análise evidenciaram que o uso de clipes com falantes nativos são os grandes potencializadores dos sentidos produzidos, o que contribui para o processo de ensino-aprendizagem de Inglês. Por fim, o estudo evidenciou divergências e similaridades quanto ao conceito de multimodalidade nas duas áreas: no Design de Interfaces, a multimodalidade corresponde à perspectiva da experiência do usuário; já na Semiótica Social, a abordagem refere-se à relação de multiplicidade dos modos envolvidos em um texto. Entretanto, o conceito de modo apresenta similaridade nas duas áreas: refere-se aos recursos e elementos visuais ou auditivos que auxiliam na materialização de sentidos.

palavras-chave:

Design de Interface; Design de Interação; Multimodalidade; Semiótica Social; Ensino de Inglês 


\section{Introdução}

O desenvolvimento tecnológico dos meios digitais propiciou a ampliação dos campos de domínio do Design, demarcados pelas áreas do Design de Interação e de Interfaces (BONSIEPE, 2011). Para Bonsiepe (2011), ambas as áreas relacionam-se com o Design de Informação, por tratarem do uso de informações e de signos. O Design de Interação trata da prática de projetar produtos digitais interativos, ambientes, sistemas e serviços com foco na criação de experiências que melhorem e ampliem a maneira como as pessoas trabalham, comunicam-se e interagem (COOPER et al., 2014; PREECE; ROGERS; SHARP, 2013; SAFFER, 2010).

Quanto à natureza do Design de Interação, é importante destacar sua característica humana, dada sua preocupação com a satisfação de necessidades e desejos das pessoas que irão interagir com um produto ou serviço, aspecto que envolve questões lógicas e emocionais (COOPER et al., 2014). Logo, o Design de Interação trata do comportamento, ou seja, como um produto afeta o comportamento humano (COOPER et al., 2014; SAFFER, 2010).

Os aplicativos são produtos digitais que possuem diversas funcionalidades e facilitam tarefas complexas, utilizando os recursos interativos dos dispositivos móveis. Além disso, os aplicativos também são utilizados como ferramentas de apoio ao ensino, possibilitando a aprendizagem móvel mobile learning ou m-learning - (CROMPTON; BURKE, 2018).

$\mathrm{Na}$ educação, o conceito de multimodalidade ganha ênfase sobretudo para apoiar a aprendizagem móvel. Para Demo (2008), a multimodalidade é uma linguagem que integra texto, som, imagem e animação - sendo o resultado do advento da tecnologia computacional (MAYER, 2001). Dessa forma, houve uma expansão na variedade dos modos de apresentação visual dos materiais, causando uma revolução no cenário da comunicação que impactou também o cenário educacional. Kress e Van Leeuwen (2006, p.17, tradução nossa) relatam que "a maioria dos textos da atualidade envolvem uma interação complexa de texto escrito, imagens e outros gráficos ou elementos sonoros, projetados como entidades coerentes por meio de um layout" - o que pode ser observado em aplicativos educacionais.

As interfaces multimodais, por sua vez, permitem uma comunicação mais exploratória e natural. Sons, gestos, vibração, fala e escrita são alguns dos recursos que podem ser utilizados para transmitir informações de variados modos. Dessa forma, as interfaces gráficas podem ser projetadas com menos recursos gráficos e mais recursos interativos, em busca de uma interação menos intrusiva (KOMNINOS, 2020).

Dentre as áreas do conhecimento em que a aprendizagem móvel é utilizada é importante destacar o ensino de línguas, devido a emergente oferta de cursos a distância por aplicativos móveis e, também, a exigência do mundo do trabalho para a aprendizagem de um segundo idioma (GOLONKA, 2014). Aprender novas línguas tornou-se uma necessidade para muitas áreas profissionais e acadêmicas, mostrando-se como um campo de pesquisa estratégico para a divulgação científica. De acordo com uma pesquisa realizada pela Education First ${ }^{1}$, apenas $5 \%$ dos brasileiros são proficientes em Inglês - o que corresponde a um nível baixo de proficiência, colocando o Brasil em $59^{\circ}$ lugar no ranking mundial (EDUCATION FIRST, 2019).

A falta de proficiente em uma língua estrangeira pode ocasionar perdas em inovação, pesquisa e tecnologia para um país. Com relação à inserção dos indivíduos no mundo do trabalho, a proficiência em um segundo idioma pode ser um diferencial no currículo, possibilitando melhores cargos e remunerações. O governo brasileiro, ciente da importância estratégica do Inglês para educação, pesquisa e inserção internacional das Universidades, desenvolveu programas para incentivar a aprendizagem dos estudantes. Em 2016, o governo ampliou as iniciativas públicas (MEC, 2017) a fim de possibilitar a mobilidade de estudantes brasileiros para outros países, fortalecendo ações em três segmentos: bolsas de intercâmbio estudantil (Programa Ciência Sem Fronteiras), exames de proficiência (apoio ao Toefl - ITP "test of english as a foreign language - integral transformative practice") e cursos de idiomas (Programa Idiomas sem Fronteiras e e-Tec Idiomas). O Ministério da

\footnotetext{
${ }^{1}$ A Education First é uma empresa de educação internacional, avaliou o nível de conhecimento nessa língua estrangeira de 1,3 milhão de adultos de 88 países, onde esse não é o idioma materno (EDUCATION FIRST, 2019).
} 
Educação (MEC), por meio da Rede e-Tec Brasil, financiou o desenvolvimento do programa nacional e-Tec Idiomas na modalidade a distância (IFSUL, 2015).

O ensino de línguas estrangeiras por meio de aplicativos vem sendo investigado na comunidade científica brasileira. Estudos recentes (ARAGÃO; LEMOS, 2017; LEFFA, 2017; MAGAL-ROYO; LABORDA, 2017; MARQUES-SCHÄFER; DA SILVA ORLANDO, 2018) descrevem os modos e recursos midiáticos e interativos utilizados por aplicativos para o ensino de línguas, entretanto não foram encontradas pesquisas que analisassem os potenciais sentidos produzidos por esses elementos.

Assim, a partir do exposto, este artigo tem como objetivo analisar como a perspectiva da multimodalidade foi adotada na interface de um aplicativo para o ensino de Inglês. Para atingir tal objetivo, foi realizada uma pesquisa de carácter exploratório, com abordagem qualitativa, envolvendo duas etapas principais: uma revisão bibliográfica e uma análise descritiva a partir dos atributos propostos por Park e Alderman (2018).

Durante a revisão bibliográfica, foi analisado o conceito de multimodalidade na Semiótica Social (HALLIDAY, 1978; HODGE; KRESS, 1988; KRESS, LEITE-GARCIA; VAN LEEUWEN, 1997; KRESS, 2010; KRESS; VAN LEEUWEN, 2001) e no Design de Interfaces (KARPOV; YUSUPOV, 2017; MULTIMODAL, 2008; OVIATT, 2003; PARK; ALDERMAN, 2018), a fim de identificar premissas para a análise do aplicativo. Após, foi realizada uma análise descritiva dos tipos de modalidade (visual, auditiva, háptica e proprioceptiva ) e dos nove atributos das multimodalidades (PARK; ALDERMAN, 2018) identificados no aplicativo. Por fim, foram discutidos aspectos relativos à potencialidade dos modos e recursos midiáticos e interativos encontrados no aplicativo que contribuem para o processo de ensino-aprendizagem de Inglês.

\section{A multimodalidade e suas Abordagens}

A multimodalidade possui relação com a multiplicidade de modos e/ou modalidades. Contudo, os conceitos de modo e modalidade possuem diferentes significados: na Linguística, remetem à Semiótica Social, para discutir a materialização de um texto; no Design, conduzem ao Design de Interfaces, a fim de relacionar os sentidos humanos com os recursos midiáticos e interativos de um dispositivo. Portanto, na revisão bibliográfica foi necessário conceituar a multimodalidade nas duas áreas que permeiam este estudo, descritas a seguir.

\subsection{A multimodalidade na Semiótica Social}

A Semiótica tem por objetivo o estudo dos modos de constituição de todo e qualquer fenômeno de produção, significação e sentido (SANTAELLA, 1983, p.1). Santaella (1983) faz uma distinção entre as linguagens verbais e não verbais, ressaltando que o século $\mathrm{XX}$ vivenciou o crescimento de duas ciências de linguagem, a saber: a Linguística - ciência da linguagem verbal - e a Semiótica - ciência de toda e qualquer linguagem.

Santaella (1983, p. 10) destaca que a língua - língua nativa, materna ou pátria - não é "a única e exclusiva forma de linguagem que somos capazes de produzir, criar, reproduzir, transformar e consumir", pois "nos comunicamos também através da leitura e/ou produção de formas, volumes massas, interações de forças, movimentos". Ainda, para a autora, "somos também leitores e/ou produtores de dimensões e direções de linhas, traços, cores [...]" (SANTAELLA, 1983, p. 10). Logo nossa comunicação também é orientada por meio de imagens, sons, gestos, toque, entre outros, pois somos "seres de linguagem" (SANTAELLA, 1983, p. 10).

A Semiótica Social, por sua vez, apropria-se do conceito de Semiótica para cobrir uma lacuna teórica que não foi evidenciada por outras abordagens. Assim, a Semiótica Social investiga as práticas humanas de "fazer significar" em circunstâncias sociais e culturais específicas, buscando explicar a criação de significados a partir da prática social. O termo foi introduzido pelo linguista Halliday (1978) no título de seu livro "Language as Social Semiotic". Para o autor, a língua é um fator social, que deve ser compreendida a partir de sua relação com a sociedade. Dessa forma, as línguas evoluem como sistemas de significados em potencial (HALLIDAY, 1978).

A multimodalidade é um desdobramento da Semiótica Social (KRESS; VAN LEEUWEN, 2001, KRESS, 2010) que busca identificar a multiplicidade de modos envolvidos em um texto. A 
Semiótica Social busca analisar o processo de produção de sentidos considerando as características inerentes à construção dos modos de comunicação (fala, escrita, imagem, entre outros) e dos recursos semióticos (gestos, tom de voz, cores, texturas, tamanhos, entre outros) presentes na materialização de um texto. A fim de contextualizar a Semiótica Social e a abordagem multimodal (KRESS; VAN LEEUWEN, 2001, KRESS, 2010), é necessário conceituar as noções de signo, texto e modo.

A unidade central da semiótica é signo, sendo ele resultado da fusão de forma e significado e presente em todos os modos. Para Kress (2010), o que distingue a Semiótica Social de outras Teorias Semióticas é a concepção de signo: na semiose da Semiótica Social, os signos são construídos nas (inter)ações sociais e somente utilizados em tais ações. Ainda, de acordo com o autor, os signos são sinais motivados e não uma relação arbitrária de significados e formas. Assim, as formas/significantes utilizadas na produção de signos são criadas na interação social e tornam-se parte dos recursos semióticos de uma cultura.

A noção de texto ultrapassa o verbal, não imitando-se aos fenômenos linguísticos de uma língua. A fala e a escrita, por exemplo, representam duas formas entre outras tantas possibilidades utilizadas para comunicar e produzir sentido (KRESS, 2010). Logo, o texto é entendido como "uma estrutura de mensagens ou de vestígios de mensagens que possuem uma unidade socialmente atribuída" (HODGE; KRESS, 1988, p.6, tradução nossa).

Já os modos são meios concretos de materializar o sentido de um texto (KRESS, 2010). Para Kress, Leite-Garcia e Van Leeuwen (1997, p. 257, tradução nossa), os textos são constituídos pela coexistência de uma ampla "variedade de modos representacionais e comunicacionais". Nesse contexto, compreende-se que sons, movimento, imagens, palavras, cores, tipografias são exemplos de modos que podem compor a materialização de um texto.

Nesta perspectiva, a Semiótica Social propõe que o verbal - texto oral ou escrito - seja compreendido por meio de uma abordagem semiótica, já que engloba um conjunto de possibilidades de modos disponíveis para a comunicação. Logo, se um texto é constituído por vários modos semióticos (KRESS, 2010), ele é multimodal.

Assim, a multimodalidade é uma abordagem que busca compreender a comunicação entre as pessoas por meio de uma variedade de formas e em situações sociais específicas. O conceito de multimodalidade surge como um desfecho da Semiótica Social, com o objetivo de investigar como os diferentes modos (KRESS; VAN LEEUWEN, 2001) relacionam-se no processo de produção e recepção dos textos.

Os modos são compreendidos como um recurso material que é utilizado como um meio de articulação de discursos - como os sons, os gestos, as imagens, entre outros. Portanto, é necessário ampliar o conceito de texto que não deve restringir-se apenas ao livro ou à página. Elementos como margens, cores, tipografias e layout são alguns exemplos dos aspectos visuais encontrados em textos escritos, o que caracteriza-os como multimodais.

Os aplicativos móveis utilizam uma variedade de modos representacionais e comunicacionais para melhorar a experiência dos usuários, tornando as interações mais intuitivas. Os recursos interativos dos dispositivos móveis permitem que as informações sejam transmitidas por meio de diferentes modos - tais como sons, gestos, vibração e voz.

Kress (2010) abordou a multimodalidade dos dispositivos móveis por meio de suas funcionalidades, a saber: captura de imagens, envio de e-mails, uso de GPS, ligações e envio de mensagens e atualização de atividades. Para o autor, a mobilidade é o elemento potencializador para o uso de cada funcionalidade; entretanto, Kress (2010, p. 109) destaca que o uso de múltiplas funcionalidades podem ocasionar a falta de atenção no uso de cada uma delas, o que compromete a multimodalidade. Logo, é necessário que o uso das funcionalidades seja atrelado aos seus potenciais sentidos.

No contexto do ensino de línguas, observa-se que os aplicativos se apropriam da multimodalidade sob viés da Semiótica Social ao utilizar diferentes modos para a memorização de palavras, verbos e expressões - o que impacta o Design de Interação e a experiência do usuário. Portanto, se faz necessário avaliar as implicações da multimodalidade no Design de Interação, temática abordada na próxima seção. 


\subsection{A multimodalidade e o Design de Interfaces}

Na interação humano-computador (IHC), as interfaces multimodais são aquelas que processam dois ou mais modos de entrada do usuário (input) de forma combinada - como a fala, o toque, o gesto e o olhar - de maneira coordenada com a saída (output) do sistema multimídia (OVIATT, 2003). As interfaces multimodais representam uma mudança de paradigma que as diferem das convencionais interfaces gráficas do usuário (MULTIMODAL, 2008).

As interfaces multimodais expandiram de forma significativa a utilização de recursos interativos (KARPOV; YUSUPOV, 2017). O input da fala, por exemplo, permite mais do que a captação da fala coloquial ou dos comandos de voz. Com o uso de sensores de áudio e vídeo, as informação não verbais do usuário - gestos, posturas, expressões faciais, emoções, caligrafia -, podem ser detectadas e analisadas. De acordo com Karpov e Yupov (2017), durante a interação humanocomputador, as modalidades são divididas em dois tipos:

- Modalidades de entrada (input): o fluxo de informações inicia do homem para o computador com métodos de entrada ativos e passivos. Na entrada ativa, o usuário transfere as informações intencionalmente - como, por exemplo, no comando de voz ou no gesto -, já na entrada passiva as informações são acompanhadas e rastreadas continuamente pelo computador - na articulação labial ou nos movimentos corporais.

- Modalidades de saída (output): o fluxo de informações vai do computador para o homem. Neste contexto, a saída multimodal também é chamada de multimídia, pois reúne as ferramentas computacionais que proporcionam a apresentação simultânea de informações heterogêneas ao usuário e os métodos de interação.

Karpov e Yupov (2017) ressaltam uma série de vantagens na utilização de interfaces multimodais, a saber: I) a naturalidade da interação humano-computador para o usuário; II) a possibilidade de entrada para informações paralelas; III) a escolha do usuário por um método conveniente de entrada/saída de informações; IV) flexibilidade de uso da interface; e V) o aumento da precisão geral da operação do sistema. As vantagens evidenciam que o uso das modalidades podem melhorar a experiência do usuário, tornando as interações com as interfaces mais naturais e flexíveis.

A relação entre a modalidade e a experiência do usuário é abordada por Park e Alderman (2018) que definem as modalidades como os padrões que moldam a forma como as pessoas utilizam os canais sensoriais para informar seus comportamentos e realizar tarefas específicas. Os autores definem a multimodalidade como o uso de vários modos ou modalidades de forma simultânea ou em sequência para afetar uma determinada ação ou atividade. Já os modos remetem aos recursos dos dispositivos que orientam a forma como eles interagem com as pessoas, com o mundo e também entre si. Dessa forma, os dispositivos utilizam os recursos para se comunicarem com as pessoas, alinhandose às modalidades humanas.

A experiência dos humanos começa pelos sentidos, sendo o único modo que possuímos de experimentar o mundo, o que nos torna seres multimodais (PARK; ALDERMAN, 2018). Por exemplo, ao nascer bebês choram espontaneamente para abrir os pulmões e expulsar o líquido que estava dentro deles, trocando-o por oxigênio; ao sentirmos cheiro de comida, nosso cérebro pode lembrar-nos que estamos com fome; o som da chuva pode significar calma ou fúria, dependendo dos nossos planos.

Compreender como os sentidos humanos funcionam é essencial para o design de novas interfaces, pois nossas interações com a tecnologia são combinadas com a forma pelas quais interagimos com o mundo físico. Park e Alderman (2018, p. 17, tradução nossa) relatam que "grande parte do Design de Interação depende de como cada sentido funciona individualmente e de habilidades que emergem de como nós os integramos", portanto mapear as modalidades sensoriais é essencial para interagir corretamente com os modos e proporcionar ao usuário uma experiência coesa. Dessa forma, é compreendido que as modalidades humanas e os modos do dispositivo necessitam atuar de forma conjunta para criar sequências de respostas que são essenciais para interações eficazes.

Park e Alderman (2018) classificam as modalidades em quatro tipos, a saber: 1) visual, baseada na visão; 2) auditiva, baseada na audição; 3) háptica, baseada no tato e movimento; e 4) proprioceptiva, baseada no deslocamento do corpo, equilíbrio, movimentação e orientação. O uso de 
duas ou mais modalidades torna uma interface multimodal. Os autores também descrevem nove atributos e habilidades das multimodalidades para fornecer premissas para o Design de Interação em produtos, explicitados a seguir.

MODALIDADES (PARK; ALDEMAN, 2018)
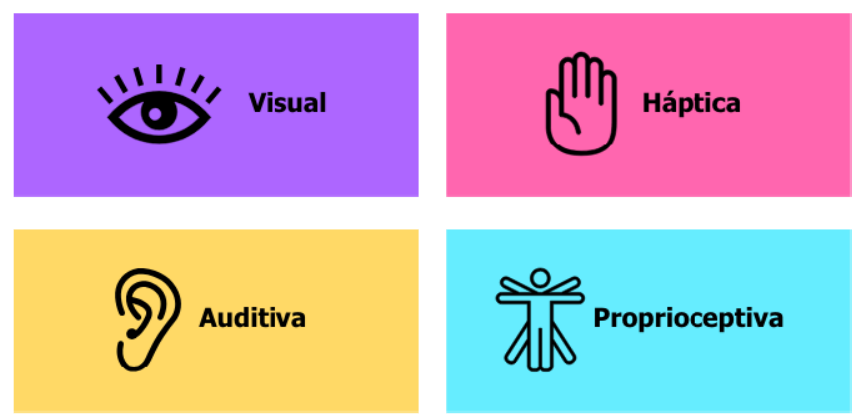

ATRIBUTOS DAS MULTIMODALIDADES

(PARK; ALERHAN, 2019)
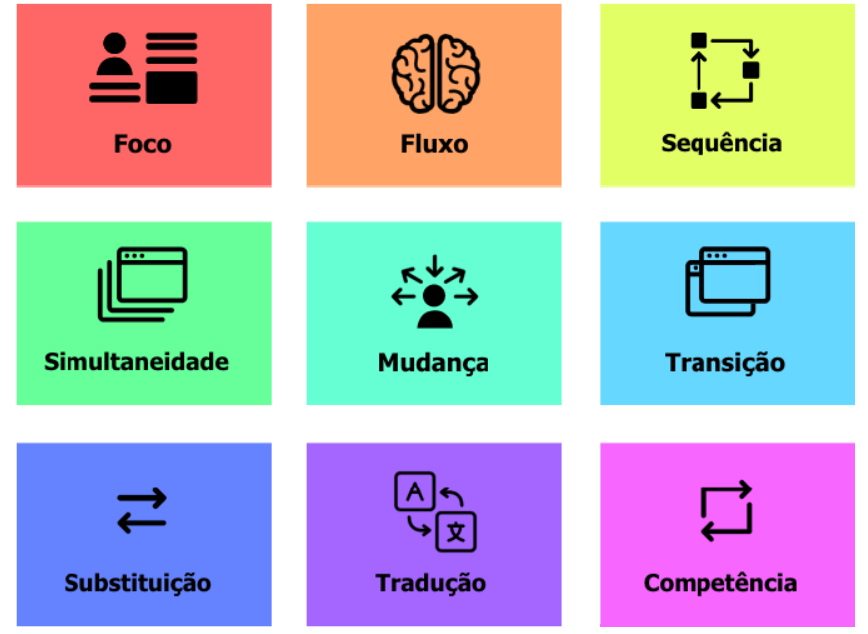

Figura 1 - Modalidades e atributos das multimodalidades. Fonte: As autoras com base em Park e Alderman (2018).

O foco faz referência à priorização das informações mais relevantes, permitindo que as tarefas possam ser concluídas com mais facilidade e eficácia. Já o fluxo é um estado da experiência em que a percepção, a cognição e a ação unem-se para otimizar o desempenho sem esforço. A sequência trata da divisão das atividades em etapas, obedecendo uma ordem específica.

A simultaneidade é a execução das atividades ao mesmo tempo. As atividades podem ser realizadas em níveis diferentes, de acordo com o foco. As mudanças ocorrem quando há alteração de um modo para outro, como por exemplo do modo visual para o auditivo. Uma mudança pode ser o resultado da progressão natural, do término de uma atividade ou causada por interrupções internas.

A transição é a maneira como as mudanças são experimentadas, podendo ser de forma leve ou inesperada. Podem ser planejadas ou não. A substituição trata da utilização de sentidos alternativos quando há interferência dentro de uma modalidade. A tradução é o mapeamento das informações para uma modalidade relevante, quando a experiência está fora da percepção humana. É também utilizada como técnica para visualização das informações e para sons de alerta. Por fim, a competência trata da repetição de atividades, fazendo com que nosso cérebro aprenda a executá-la sem pensar. Dessa forma, a atividade pode ser realizada com pouca ou nenhuma consciência.

Nas interfaces multimodais, os usuários possuem preferências distintas entre as modalidades. Em uma atividade, por exemplo, algumas pessoas consideram-se melhores na compreensão da linguagem por meio da leitura e da escrita, enquanto outras preferem ouvir ou dialogar. Logo, ainda 
que seres humanos sejam multimodais, existem preferências baseadas nas experiências individuais dos usuários. Portanto, os atributos apresentados devem ser considerados para o Design de Interfaces multimodais, sobretudo para a aprendizagem móvel.

A aprendizagem móvel tem sido amplamente utilizada no ensino de línguas, dada a extensa oferta de cursos por aplicativos. Na atualidade, os aplicativos para a aprendizagem de línguas são projetados para desencadearem experiências de ensino e aprendizagem autodirecionadas e holísticas (EISENLAUER, 2014). A qualidade da interface gráfica dos aplicativos aliada as modalidades propiciadas por meio de recursos interativos - tais como gravação de voz, reprodução de sons e vídeos, notificações push, feedback automático - possibilitam diversas opções para o aprimoramento das habilidades linguísticas de um aluno.

O aplicativo Memrise, por exemplo, busca engajar os usuários na aprendizagem de línguas por meio de vídeos curtos de falantes nativos de cada língua, com suas vozes e sotaques autênticos, além da percepção sobre cada religião e cultura. Entretanto, como a perspectiva da multimodalidade foi adotada na interface de um aplicativo para o ensino de inglês? A fim de responder esse questionamento, foi realizada uma análise do aplicativo Memrise, apresentada a seguir.

\section{Análise descritiva}

A análise, de carácter descritivo e exploratório, teve como objetivo identificar os modos e os recursos midiáticos e interativos com base nos tipos de modalidade (visual, auditiva, háptica e proprioceptiva) e nos atributos das multimodalidades (PARK; ALDERMAN, 2018) identificados no aplicativo. Dessa forma, foi possível analisar como a perspectiva da multimodalidade foi adotada na interface de um aplicativo para o ensino de Inglês. Logo, mais do que identificar modos e modalidades utilizados pelo aplicativo para o ensino de Inglês, buscou-se relacionar a produção de sentidos produzida pelos textos multimodais utilizados no aplicativo que se manifestam por meio de imagens, sons, cores, layout, entre outros elementos.

A análise foi organizada em três momentos: primeiramente, foi realizada uma breve contextualização do aplicativo selecionado; segundamente, foram analisados os tipos das modalidades e atributos das multimodalidades (PARK; ALDERMAN, 2018) e, por fim, foi utilizada a abordagem da multimodalidade da Semiótica Social (KRESS, 2010) para descrever os modos e recursos midiáticos e interativos identificados.

O aplicativo Memrise, disponibilizado para download desde 2013, possui mais de 42 milhões de usuários em 189 países. Diferente de outros aplicativos populares para o ensino de línguas - como o Duolingo, busuuu, Babel -, o Memrise utiliza clipes de falantes nativos de cada língua para ensinar as palavras e frases do curso. Dessa forma, o aluno aprende com as vozes e sotaques autênticos de cada lugar, além de obter uma percepção sobre a cultura de cada região. O Memrise organiza a aprendizagem de línguas em níveis, subdividas em unidades, compostas por exercícios variados. Para a análise, foi considerada a primeira unidade de inglês (americano 1) para iniciantes do nível 1.

O nível 1, intitulado "Palavras e frases - Base de lançamento", tem como objetivo ensinar 30 palavras ou frases. Ao selecionar um dos itens, o aluno visualiza um clipe de um falante nativo pronunciando a palavra e/ou frase. É importante destacar que, ao repetir a mesma palavra, o clipe é alterado: o sujeito e o cenário são outros, mas a palavra e/ou frase pronunciada é a mesma. A Figura 2 apresenta a sequência de interações descritas anteriormente. Ao selecionar a palavra "hi", o usuário é redirecionado para a interface contendo o clipe do falante nativo (uma mulher sentada em frente a uma casa), logo abaixo, a tradução da palavra para a língua do usuário e uma área para adicionar notas ou lembretes $(\mathrm{Mems})$ que possa auxiliar o usuário a memorizar a palavra. Se o usuário quiser repetir a palavra, ele visualizará um clipe de outro falante nativo, representado na Figura 2 por um homem em um ambiente externo. 


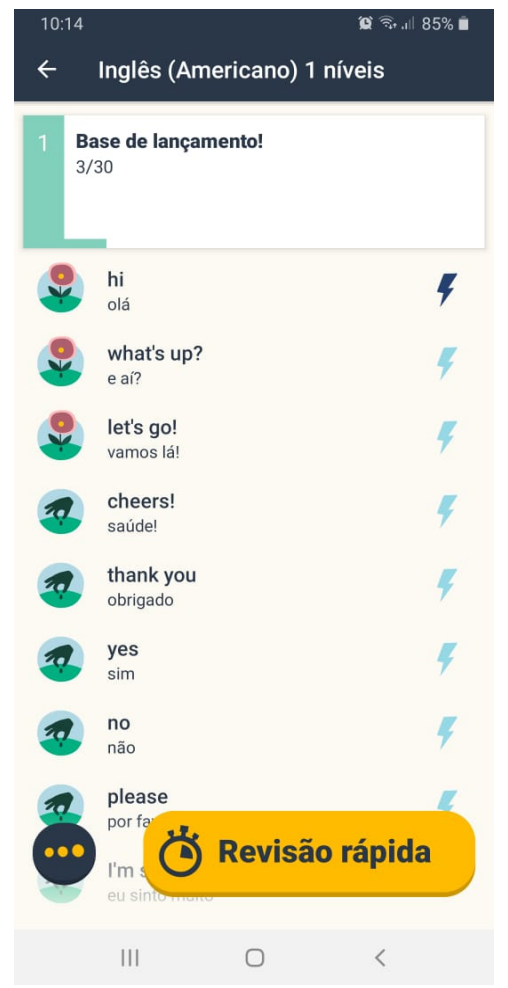

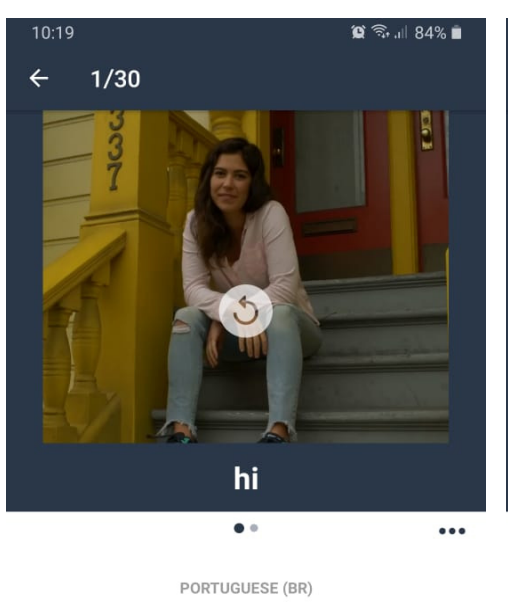

olá

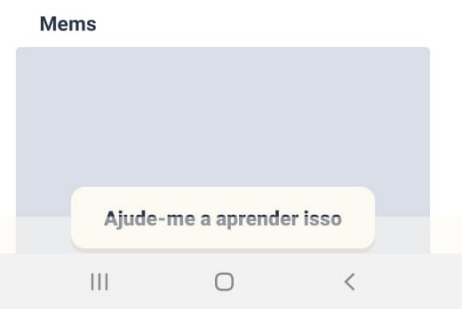

Figura 2 - Nível 1 (Palavra "Hi”). Fonte: Aplicativo Memrise.

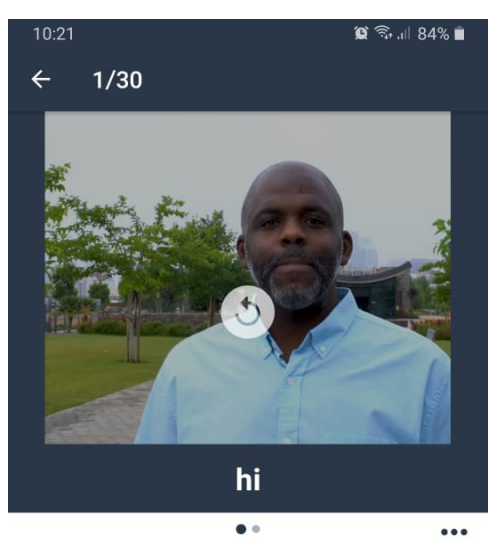

PORTUGUESE (BR)

olá

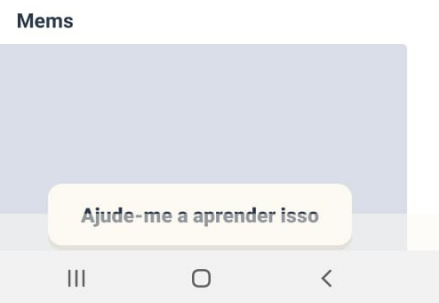

Com relação às modalidades utilizadas pelo aplicativo, foram identificadas o uso da visual e da auditiva, o que torna a interface multimodal (PARK; ALDERMAN, 2018). Durante todas as atividades, o usuário necessita visualizar e ouvir o clipe para memorizar as palavras e frases. Isso também acontece na "Revisão rápida", disponível na Figura 2, em que o usuário realiza uma série de exercícios para memorização de vocabulário. O resultado da análise realizada a partir da identificação dos nove atributos e habilidades das multimodalidades propostos por Park e Alderman (2018) pode ser observada no Quadro 1.

\begin{tabular}{ll|l|l|l}
\hline \multicolumn{1}{c|}{ ATRIBUTO } & SIM & $\begin{array}{c}\text { EM } \\
\text { PARTE }\end{array}$ & NÃo \\
\hline 1. Foco & & & \\
\hline 2. Fluxo & & & \\
\hline 3. Sequência & & & \\
\hline 4. Simultaneidade & & & \\
\hline 5. Mudança & & & \\
\hline 6. Transição & & & \\
\hline 7. Substituição & & & \\
\hline 8. Tradução & & & \\
\hline 9. Competência & & & \\
\hline
\end{tabular}

Quadro 1 - Nove Atributos e Habilidades das Multimodalidades identificados no App Menrise. Fonte: As autoras com base em Park e Alderman (2018). 
A partir do Quadro 1 observa-se que o Memrise contempla boa parte dos atributos das multimodalidades, destacando-se o foco, o fluxo, a simultaneidade e a transição. Esses atributos atuam em conjunto, auxiliando o usuário em seu processo de aprendizagem, como pode ser observado nas atividades dos itens "Aprender" e "Revisar", conforme Figura 3. Os itens contemplam exercícios de revisão do tipo múltipla escolha, escrita e fala. Todos os exercícios utilizam duas modalidades - a visual e a auditiva - de forma que uma é dependente da outra, ou seja, o usuário precisa visualizar e ouvir o clipe e/ou áudio para selecionar a alternativa correta ou escrever a resposta, como no exemplo da Figura 3.

$\mathrm{O}$ atributo foco é identificado na hierarquia dos elementos da interface, o que facilita a interação e a compreensão sobre as atividades e lições. O fluxo pode ser verificado nos exercícios do item “Aprender - Palavras e Frases", disponível na Figura 3, em que a percepção, a cognição e a ação são utilizadas em conjunto para realizar a atividade sem sobrecarga cognitiva. Ainda, na Figura 3, é possível identificar o uso da simultaneidade pela utilização das duas modalidades - a visual e a auditiva - para a realização das atividades. Foi também identificado o uso de transições leves e planejadas, ocorrendo entre as interfaces de navegação do aplicativo.
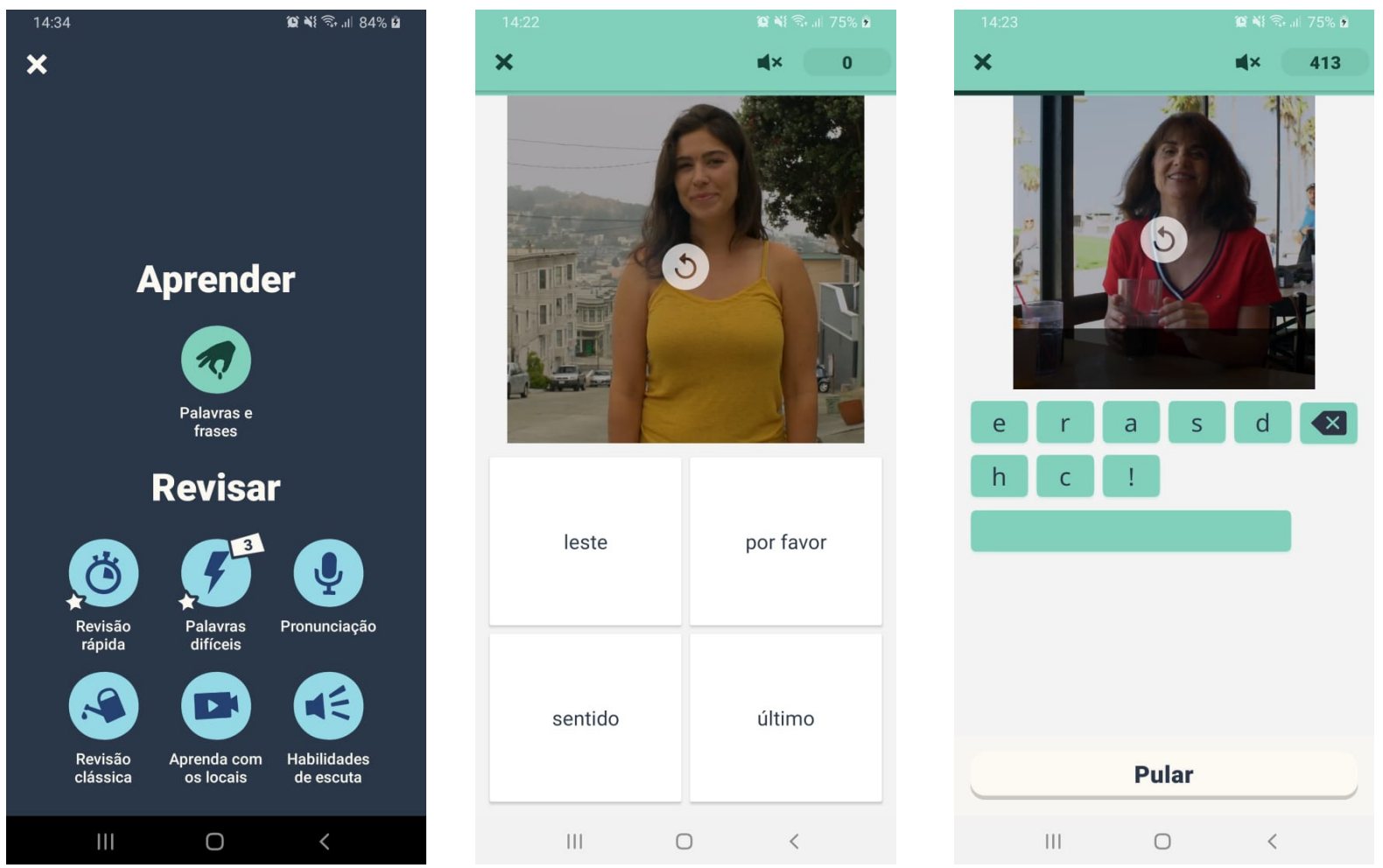

Figura 3 - Atividades do item Aprender ("Palavras e Frases")

Fonte: Aplicativo Memrise.

Com relação à sequência, foi constatado que embora o aplicativo organize a aprendizagem em diferentes níveis (Figura 2), o aspecto randômico na qual as atividades dos itens "Aprender" e "Revisar" são organizadas pode prejudicar a compreensão, conforme exemplificado na Figura 4. No nível 1, por exemplo, são exibidas as palavras e frases para serem memorizadas. No rodapé observa-se um botão, com a chamada "Palavras e frases", que redireciona o usuário para um revisão contendo as palavras e frases acompanhadas do clipe intercaladas com exercícios de múltipla escolha. O botão de chamada "Palavras e frases" é randômico, ou seja, ao retornar para o menu principal do nível 1 ele redireciona para outras revisões, chamadas de "Revisão clássica", "Aprenda com os locais" ou "Habilidades de escuta". Dessa forma, o aluno não sabe exatamente quais atividades estarão disponíveis e nem pode selecionar qual habilidade quer exercitar, o que pode comprometer a sequência de aprendizagem. 

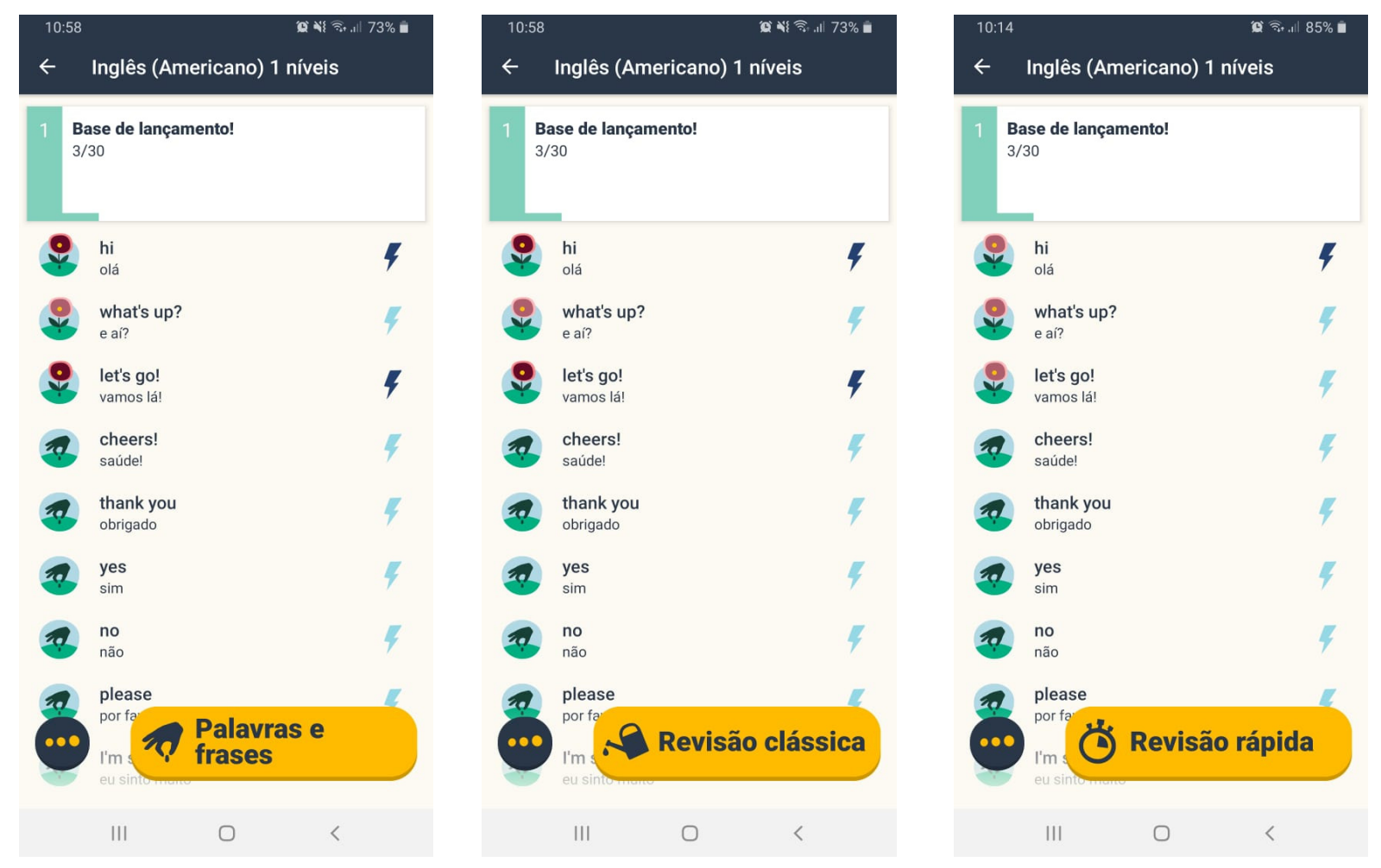

Figura 4 - Atividades do item Aprender ("Palavras e Frases")

Fonte: Aplicativo Memrise.

O atributo da mudança também foi analisado como em parte, pois durante a aprendizagem de frases e palavras não existem mudanças repentinas de interação: o usuário apenas visualiza o clipe, a tradução para sua língua e a tradução literal, sendo a única interação possível a escrita no item "Mems". Entretanto, em "Aprender" e "Revisar" o usuário realiza uma série de atividades que utilizam as duas modalidades de forma intercalada, conforme explicitado anteriormente.

A substituição não foi adotada pelo aplicativo, tendo em vista que não foi observada a utilização de sentidos alternativos quando houve interferência em uma modalidade. Por se tratar de um aplicativo para o ensino de línguas, é compreensível que ele utilize as modalidades sem interferência.

A tradução é utilizada em sua forma literal: ela acompanha os clipes dos falantes nativos, contendo a tradução para a língua nativa do usuário. Por fim, a competência é um atributo muito explorado pelo aplicativo, dado o seu objetivo: a memorização de vocabulário. Todos os exercícios exploram a repetição e a memorização.

\section{Discussão}

A análise descritiva do aplicativo Memrise, guiada pelos tipos de modalidade e atributos das multimodalidades de Park e Alderman (2018) e pela abordagem multimodal de Kress (2010), permitiu identificar aproximações entre o conceito de multimodalidade no Design e na Semiótica Social. A interface do aplicativo, com seus recursos midiáticos e interativos, pode ser compreendida como um texto integrado. Por exemplo, na Figura 3, o significado da palavra "please" é compreendido pela entonação e contexto do sujeito no clipe, o que auxilia no processo de memorização. Da mesma forma, o significado da palavra "cheers" pode ser entendido pela relação entre o sujeito e seu entorno: uma mulher em um restaurante, sentada em uma mesa e movimentando um copo.

Com relação às modalidades utilizadas no aplicativo, foram identificadas duas, a saber: a visual e a auditiva. Todavia, o aplicativo poderia também utilizar a modalidade háptica por meio do recurso de vibração dos dispositivos móveis, atrelando-a ao feedback de erro em um exercício, por exemplo. 
Outro aspecto a ser destacado é com relação a variação de sujeitos utilizados nos clipes, como explicitado anteriormente. No aplicativo Memrise, os sujeitos não são apenas alegóricos: eles são personagens inseridos em contextos reais que auxiliam o usuário em seu processo de aprendizagem. Os sujeitos dos clipes aparecerem em diferentes níveis, inseridos em outros contextos, mas seguem estabelecendo o contato visual com o usuário, falando diretamente para ele. A variação das vozes, das entonações e dos sotaques podem possibilitar que o usuário desenvolva melhores habilidades auditivas, tornando-o capaz de identificar a pronúncia com suas variações. É importante destacar que muitos aplicativos para o ensino de línguas utilizam somente duas vozes - uma feminina e outra masculina - para ditar as palavras ou frases e que, muitas vezes, as vozes são mecanizadas.

No Memrise foram identificados diferentes modos (KRESS; LEITE-GARCIA; VAN LEEUWEN, 1997; KRESS; VAN LEEUWEN, 2001; KRESS, 2010), tais como o vídeo, o áudio, o texto, o layout, os gestos (protagonizados pelos sujeitos nos clipes), a fala, entre outros. Dentre os modos indicados, foi percebido que os vídeos - chamados de clipes pelos produtores do aplicativo são os grandes potencializadores dos sentidos produzidos. Os vídeos são o diferencial competitivo do Memrise quando comparado com outros aplicativos para o ensino de línguas. Eles podem ainda possibilitar uma conexão emocional com o usuário, o que pode auxiliá-lo no processo de aprendizagem. Ao invés de memorizar a palavra e/ou frase por meio de uma imagem estática, o usuário poderá memorizar o falante nativo no clipe.

O Memrise utiliza uma variedade de modos, visando explorar os recursos midiáticos e interativos dos dispositivos móveis para a aprendizagem de Inglês. Dentre os modos observados, o vídeo foi o que demostrou apresentar maior potencial de sentido por articular os diferentes modos em uma única mídia: a imagem, o áudio e o texto foram contemplados pelo vídeo. É importante destacar que o potencial de sentido apresentado pelo vídeo ocorre devido a sua materialização, ou seja, os clipes com falantes nativos foram produzidos com um objetivo concreto: fazer a relação entre a palavra e/ou frase e o contexto de uso.

As atividades propostas pelo aplicativo utilizam duas modalidades - a visual e a auditiva - e exploram diferentes modos, representados por diferentes arranjos de layout. Os vídeos apresentam novas palavras e frases, sendo também utilizados para ilustrar o enunciado de exercícios, ao invés das convencionais representações textuais.

\section{Considerações finais}

O presente artigo se propôs a analisar como a perspectiva da multimodalidade foi adotada na interface de um aplicativo para o ensino de Inglês. Para isso, foi necessário recorrer a um referencial teórico que tratasse da abordagem multimodal a partir da Semiótica Social, mais especificamente da Linguística, e sua relação com o Design de Interfaces. Dessa forma, foi possível evidenciar como as práticas do Design potencializam os processos de aprendizagem, como o ensino de Inglês.

O campo do Design apresenta a multimodalidade na perspectiva da experiência do usuário, compreendendo-o como um ser humano multimodal. Já a Semiótica Social discute a multimodalidade para analisar a multiplicidade de modos envolvidos em um texto. Logo, foi identificada uma divergência com o conceito de multimodalidade.

Ambas áreas tratam do conceito de modo: no Design, o termo refere-se aos recursos dos dispositivos que orientam a forma como eles interagem com as pessoas, com o mundo e também entre si (PARK; ALDERMAN, 2018); na Semiótica Social, o conceito remete aos meios concretos de materializar o sentido de um texto (KRESS, 2010). Ainda, na Semiótica Social, os modos são representados por sons, movimento, imagens, palavras, cores, tipografias. Logo, é possível perceber uma similaridade entre as duas abordagens com relação ao conceito de modo, o que pode ser identificado na análise do aplicativo.

No presente estudo foram investigadas as duas perspectivas da multimodalidade, o que resultou em uma análise com maior profundidade. Enquanto o Design compreende o conceito de multimodalidade como utilização de vários modos ou modalidades para ação, a Semiótica Social investiga sobre a multiplicidade de modos de comunicação envolvidos na materialização de um texto. Logo, a multimodalidade no Design foi utilizada como um guia para a análise e a abordagem da Semiótica Social auxiliou na descrição dos elementos observados. 
A fim de analisar a percepção dos usuários sobre a perspectiva da multimodalidade no aplicativo, estudos subsequentes irão envolver testes com usuários por meio de diários narrativos, em que se pretende discutir a efetividade das modalidades assumidas pelo Memrise.

The multimodality perspectives in the analysis of modes and resources of an application for teaching English

Abstract: Multimodal interfaces allow more exploratory and natural communication. It provides users several modes of interaction. In education, multimodality refers to the use of different modes of communication to enhance the teaching-learning processes of reading and writing, especially in the teaching of foreign languages. Thus, this article proposes to analyze how the multimodality perspective was used in the interface of an application for teaching English, according to the multimodal approach in the Interface Design and Social Semiotics. Therefore, it was realized exploratory research, with a qualitative approach, based on a literature review and a descriptive analysis. The results showed that the use of clips with native speakers is the great enhancer of the produced meanings, which contributes to the teaching-learning processes of English. Finally, the study identified divergences and similarities towards the multimodality concept in the two areas: in Interface Design, multimodality corresponds to the perspective of the user experience; on the other hand, in Social Semiotics the approach refers to the multiplicity of modes involved in a text. However, the mode concept has similarities in the two areas: it refers to the resources and/or visual or auditory elements that help in the meaning's materialization.

Keywords: Interface Design; Interaction Design; Multimodality; Social Semiotics; English Teaching.

\section{Referências bibliográficas}

ARAGÃO, R.; LEMOS, L. WhatsApp e multiletramentos na aprendizagem de inglês no Ensino Médio. In: Polifonia, v. 24, n. 35/1, p. 73-94, 2017. Disponível em:

<https://periodicoscientificos.ufmt.br/ojs/index.php/polifonia/article/view/6034/>. Acesso em: 9 ago. 2020.

BONSIEPE, G. Design, Cultura e Sociedade, Blucher. São Paulo: 2011.

COOPER, A.; REIMANN, R.; CRONIN, D.; et al. About face: the essentials of interaction design, John Wiley \& Sons. Indianapólis: 2014.

CROMPTON, Helen; BURKE, Diane. The use of mobile learning in higher education: A systematic review. In: Computers \& Education, v. 123, p. 53-64, 2018.

DEMO, P. Os desafios da linguagem do século XXI para a aprendizagem da escola. Palestra, Faculdade OPET, Curitiba, junho de 2008. Disponível em:

<https://caldeiraodeideias.wordpress.com/2009/08/05/pedro-demo-aborda-os-desafios-da-linguagemno-seculo-xxi/>. Acesso em: 08 jun. 2020.

EDUCATION FIRST (EF). Índice de Proficiência em Inglês da EF. 2019. Disponível em: <https://www.ef.com.br/epi/>. Acesso em: 10 ago. 2020.

EISENLAUER, V. Multimodality in mobile-assisted language learning. In: Communications in

Computer and Information Science, [S. I.], v. 479, p. 328-338, 2014. Disponível em: <https://doi.org/10.1007/978-3-319-13416-1_32/>. Acesso em: 21 jul. 2010. 
GOLONKA, E.M. et al. Technologies for foreign language learning: a review of technology types and their effectiveness. In: Computer assisted language learning, v. 27, n. 1, p. 70-105, 2014.

HALLIDAY, M. A. K. Language as social semiotic: The social interpretation of language and meaning, University Park Press. Maryland: 1978.

HODGE, R., KRESS, G. Social Semiotics, Polity Press. London: 1988.

INSTITUTO FEDERAL SUL-RIO-GRANDENSE (IFSUL). Núcleo de Idiomas - e-Tec Idiomas.

Brasília, 2015. Disponível em: <http://www.ifsul.edu.br/nucleo-de-idiomas-ifsul/e-tec-idiomas/>. Acesso em: 08 ago. 2020.

KARPOV, A. A.; YUSUPOV, R. M. Multimodal Interfaces of Human-Computer Interaction. In: Herald Of The Russian Academy Of Sciences, [S.L.], v. 88, n. 1, p. 67-74, jan. 2018. Disponível em: <https://link.springer.com/article/10.1134/S1019331618010094\#citeas/>. Acesso em: 10 ago. 2020.

KOMNINOS, A. No-UI: how to build transparent interaction. How to Build Transparent Interaction. 2020. In: Interaction Design Foundation. Disponível em: <https://www.interaction-

design.org/literature/article/no-ui-how-to-build-transparent-interaction/>. Acesso em: 21 jul. 2020.

KRESS, G. R. Multimodality: A social semiotic approach to contemporary communication, Taylor \& Francis. New York: 2010.

KRESS, G.; LEITE-GARCIA, R.; VAN LEEUWEN, T. Discourse Semiotics. In: VAN DIJK, T. Discourse as structure and process, Sage Publications. New Delhi: 1997.

KRESS, G.; VAN LEEUWEN, T. Multimodal discourse: the modes and media of contemporary communication. Hodder Arnold. London: 2001.

LEFFA, Vilson J. Produção de materiais para o ensino de línguas na perspectiva do design crítico. Construções de sentido e letramento digital crítico na área de línguas/linguagens, Pontes Editores. Campinas: 2017. Disponível em:

<http://www.leffa.pro.br/textos/trabalhos/design_critico.pdf/>. Acesso em: 9 ago. 2020.

MAGAL-ROYO, T.; LABORDA, J. G. Multimodal interactivity in foreign language testing. In:

Multimodal Interaction with W3C Standards, Springer. Cham: 2017. Disponível em: < https://link.springer.com/chapter/10.1007/978-3-319-42816-1_16/>. Acesso em: 9 ago. 2020.

MARQUES-SCHÄFER, G.; DA SILVA ORLANDO, A. A. Concepções de aprendizagem de línguas e o Duolingo: uma análise crítica sobre sua proposta e experiências de aprendizes. In: Texto Livre: Linguagem e Tecnologia, v. 11, n. 3, p. 228-251, 2018. Disponível em:

<http://www.periodicos.letras.ufmg.br/index.php/textolivre/article/view/14292/>. Acesso em: 9 ago. 2020.

MAYER, R. E. Multimedia learning, Cambridge University Press. Cambridge: 2001.

MINISTÉRIO DA EDUCAÇÃO (MEC). Idiomas sem Fronteiras (IsF). Brasília, 2017. Disponível em: <http://isf.mec.gov.br/>. Acesso em: 08 ago. 2020.

MULTIMODAL Interfaces. In: FURHT, Borko (ed.). Encyclopedia of Multimedia, Springer. Boston: 2008. p. 650-659. Disponível em: <https://link.springer.com/referenceworkentry/10.1007\%2F978-0387-78414-4_159/>. Acesso em: 10 ago. 2020.

OVIATT, S.L. Multimodal Interfaces. In: JACKO, J.; SEARS, A. (ed.). The human-computer interaction handbook: fundamentals evolving technologies and emerging applications, Lawrence Erlbaum. New Jersey: 2003. Cap. 14. p. 286-304.

PARK, C. W.; ALDERMAN, J. Designing across senses: a multimodal approach to product design, O'Reilly Media, Inc. Sebastopol: 2018.

PREECE, J.; ROGERS, Y.; SHARP, H. Design de Interação: além da interação homem-computador, Bookman. Porto Alegre: 2013.

SAFFER, D. Designing for interaction: creating innovative applications and devices, New Riders. Berkeley: 2010.

SANTAELLA, L. O que é semiótica? Editora Brasiliense. São Paulo: 1983. 